A.A. Krivoshapkina, A.I. Subbotovskaya, O.V. Strunin, A.N. Shilova, A.A. Efimov, V.S. Kozyreva, I.A. Kornilov

Meshalkin Novosibirsk research institute of circulation pathology of the Ministry of Health of the Russian Federation

\title{
Neutrophil CD64 index in sepsis diagnosis after cardiac surgery in infants
}

\section{Author affiliation:}

Krivoshapkina Aleksandra Alekseevna, junior research scientist at the group of pediatric anesthesiology and resuscitation science of the Center of pediatric cardiosurgery and neonatal surgery of the Meshalkin Novosibirsk research institute of circulation pathology (Federal State Budgetary Institution) of the Ministry of Health of the Russian Federation.

Address: 15, Rechkunovskaya Str., Novosibirsk, 630050; e-mail: paleksandra@mail.ru

Article received: 14.01 .2014 . Accepted for publication: 14.05 .2014 .

Early diagnosis of sepsis in neonates is especially relevant due to the persistently high rate neonatal mortality caused by this pathology. Active search for new laboratory markers, which would help to predict onset of a generalized infectious process with high probability, has been going on in recent years. The study was aimed at determining sensitivity and specificity of the CD64 index, evaluating the possibility of using it as an early marker of sepsis in infants after cardiac surgery during artificial circulation and comparing prognostic value of the CD64 index and the routinely used parameters. Patients and methods: the study involved 36 postoperative ICU. Retrospective analysis of bacterial inflammation markers has been conducted in groups of children at the postoperative stage: with $(n=16)$ and without $(n=20)$ sepsis. Results: the CD64 index in patients with sepsis was significantly higher than in the children without sepsis$2.29(p=0.01)$. At the same time, blood serum $C$-reactive protein concentration and the absolute peripheral blood leukocyte count were similar in patients from both groups. Procalcitonin (PCT) test sensitivity for sepsis diagnosis was $65 \%$, specificity - 67\%. At the same time, the negative prognostic value of PCT reached $76 \%$, the positive one - 63\%. The performed analysis demonstrated that the CD64 index is a reliable early marker of sepsis with high sensitivity (80\%) and specificity (90\%); AUC-0.895 (0.753-1.000) at cut-off point 2.00. Conclusions: C-reactive protein may not be seen as a reliable early marker of sepsis due to, it appears, peculiarities of its dynamics in the postoperative period. Specificity of this parameter is low and amounts to 50\%. The study also demonstrated lower information value of the procalcitonin test compared to the CD64 index. The CD64 index over 2.00 indicates high risk of sepsis development in neonates in the postoperative period.

Keywords: CD64 index, sepsis, neonates, infants, procalcitonin, cardiac surgery, cardiopulmonary bypass.

Nowadays, the problem of sepsis in infants and neonates is a global one [1]. According to the World Health Organization and UNICEF, more that 20\% of deaths of neonates in 2013 were caused by sepsis. Despite wide research and improvements in management of sepsis, it still remains the principal cause of morbidity and mortality at neonatal intensive care units, and its prevalence is constantly growing [2].

The main issue in diagnosing sepsis lies in the fact that its clinical manifestations could resemble those of SIRS (systemic inflammatory response syndrome), for instance, small cardiac output, respiratory distress syndrome, tissue damage, acute adrenal gland deficiency etc. in infants and neonates [3]. Diagnosing sepsis is especially important for both neonates and infants with congenital heart diseases, who were previously operated with the use of cardiac bypass, which, in itself, may cause systemic inflammatory response [4]. Evaluation of severity and early 
diagnosis of sepsis in these patients pose a serious problem due to peculiar nature of postoperative period. It is often very difficult to determine the cause of deterioration in the condition of a patient and development of multiple organ failure. Differentiating between sepsis, systemic inflammatory response and progressive cardiopulmonary disorders with consecutive multiple organ failure without infection also makes for a complex problem.

Not only bacteria, but viruses and fungi could serve as the pathogens triggering that process. To confirm the diagnosis, in many cases a hemoculture positive for sepsis is needed. However, the fact that, due to small amounts of blood taken, culture growth can be observed only in $30 \%$ of the cases has to be taken into consideration. Acquisition of results of microbiological analysis often takes a large amount of time and could postpone diagnosis establishment and beginning of treatment $[4,5]$.

Specialists are still looking for new markers of sepsis that would allow diagnosing the condition at the earliest stages possible (before the development of entire clinical pattern) or predicting its forthcoming development with high probability rate. Early diagnosis of sepsis and timely and adequate antibacterial treatment sufficiently influence clinical outcomes in a positive way.

Specialists have not yet found a single marker that is equally informative for neonates and infants among the routine laboratory tests. For example, C-reactive protein (CRP) routinely used for diagnosing bacterial sepsis in neonates and infants cannot be definitively called an early stage marker due to its low specificity $[6,7]$. Procalcitonin is used for diagnosing bacteremia in infants more often than CRP [8], yet it produces small informative value in neonates due to its physiologically high level during first days of life [9]. Therefore, the problem of finding new early stage diagnostic markers for sepsis that can be applied to treatment of different age groups of children regarding differentiation between sepsis and other infectious and non-infectious processes accompanied by systemic inflammatory response remains a huge challenge.

Currently, the usage of neutrophil expression level of Fc $\gamma \mathrm{RI}$ (CD64) receptor for early diagnosis of sepsis in different categories of patients is under consideration. Some authors point to the fact that during the development of sepsis the neutrophil expression level of CD64 undergoes significant increase. Therefore, we may assume that the variability of neutrophil expression of Fc $\gamma$ RI (CD64) receptors could potentially serve as a bacterial sepsis marker. Other authors demonstrated the possibility to use it as an early differential criterion of sepsis in adults [10-15], neonates [14-16] and infants [17]. Currently, no data from cardiac surgery units are available; also, no data are available on the possibility of using this parameter as a sepsis marker in infants after cardiosurgical interventions employing cardiac bypass, because all of the studies on this subject were carried out at general intensive care units.

This pilot study was aimed at determining sensitivity and specificity of the CD64 index, evaluating its applicability as an early marker of sepsis in the infants that have undergone cardiosurgical interventions employing cardiac bypass and comparing its prognostic value to the routinely used parameters.

\section{PATIENTS AND METHODS}

This study was carried out at the Meshalkin Novosibirsk research institute of circulation pathology of the Ministry of Health of the Russian Federation in May-September 2013. 36 children were being postoperatively monitored at the resuscitation and intensive care unit (RICU), 16 of whom were neonates ( $<28$ days of age); 20 children aged from 28 days to 12 months. All the patients have previously undergone cardiac bypass surgery to treat congenital heart disorders. Nosologic classification of the patients is given in tb. 1 .

Markers of bacterial inflammation in venous blood were analyzed at suspicion of potential sepsis/SIRS development. On the average, blood samples were taken on postoperative day 2 or 3. To determine the CD64 index, procalcitonin and CRP values, the whole EDTA-stabilized venous blood samples were analyzed. 
The study involved patients with SIRS clinical pattern, with a possible infectious process or with clinical pattern of both SIRS and infectious process.

SIRS and sepsis diagnosis was carried out on the basis of the criteria approved by the International Joint Pediatric Conference on sepsis in 2002 [5]. Sepsis was defined as a systemic inflammatory response either emerging from infection or confirmed by microbiological analysis or in the presence of other clinical manifestations of infection. The SIRS criteria are given in tb. 2 .

Suspicion of infection or SIRS was defined as a direct mention made by an emergency doctor in the respective patient's clinical chart on the basis of the aforementioned criteria; the mention of suspicion of onset of the infection associated with prolonged artificial pulmonary ventilation (APV) or infeasibility of removing the patient from the ventilator; deterioration of condition of children with an initially unfavorable condition (repeated operative interventions, intrauterine infection in the anamnesis, pulmonary alterations [radiography], surgical sternal diastasis or delayed sternotomic wound closure). Upon inclusion of patients into the study, several other factors were taken into consideration as well, including results of microbiological assays of blood and physiological secretions, as well as routine laboratory tests, such as white blood count, leukogram, chest radiography etc., that could point to a possible infection.

Retrospectively, after having been released from the RICU, the patients were divided into 2 groups: with or without sepsis.

Diagnosis of bacterial, fungal or viral sepsis was confirmed with positive bacterial/fungal blood culture, positive polymerase chain reactions in blood sample assay, serological tests, positive culture of urine, tissue secretions and tracheal aspirate. Sepsis was also diagnosed in several patients with blood culture negative for sepsis on the basis of clinical manifestations of SIRS and pronounced antibacterial treatment effectiveness.

Procalcitonin (PCT) was determined through the use of semiquantitative immunochromatographic test by means of dipsticks Brahms PCT-Q (Thermo Scientific, Germany). CRP levels were measured using automated biochemical analyzer AU 680 (Beckman Coulter, USA). Neutrophil CD64 index was defined according to the manufacturer's recommendations provided with test system Leuko 64 (Beckman Coulter, USA) using flow cytofluorimeter Navios (Beckman Coulter, USA).

\section{Statistical analysis}

The data were statistically manipulated using program SPSS v.20.0 (SPSS Statistics, USA). All data samples were checked for normal distribution using Shapiro-Wilk test. Due to asymmetric distribution of values of parameters, the median (M) and quartiles (Q25-Q75) were used to describe the data. Differences between groups were evaluated on the basis of non-parametric Mann-Whitney test for independent samples. To compare qualitative indicators, chi-square test was used. Diagnostic features of bacterial inflammation markers were evaluated using ROC (receiver operator characteristic) analysis in order to determine sensitivity (share of the true positive results) and specificity. The point of the ROC curve with the largest sum of sensitivity and specificity was taken as an interval parameter value. To assess information value of semiquantitative indices of the PCT test, the likelihood ratio as well as the odds ratio (OR) and confidence intervals (CI) thereof were calculated. Values at $\mathrm{p}<0.05$ were considered statistically significant.

\section{RESULTS AND DISCUSSION}

16 out of 36 neonates and infants developed sepsis (diagnosis was confirmed by positive blood culture in 3 of them). 21 children other were sepsis-free ( 9 of them had only SIRS; 8 - other infectious complications without the generalized process; 3 had cardiopulmonary failure, which 
was primarily associated with the initial severity of the disorder and surgical intervention and no infectious complications in the postoperative period).

Clinical data and level of bacterial inflammation markers were analyzed in two groups of patients - with and without sepsis (tb. 3).

Patients of the two groups were comparable in terms of age and such parameters of the undergone operative intervention as cardiac bypass duration and duration of aortic occlusion. Those children who developed sepsis remained significantly longer at the intensive care unit, required longer artificial pulmonary ventilation and inotropic support. We also observed a higher mortality rate in that group (see tb. 3 ).

The CD64 index in the patients with sepsis amounted to 2.29 (Q1-Q3 - 1.96-3.32), i.e. significantly higher than in the children without sepsis $(\mathrm{p}=0.001)$. At the same time, the CRP concentration in blood serum and the absolute peripheral white blood count were identical in the two groups (see tb. 3).

Features of the test system used to determine the procalcitonin level help to differentiate between the following ranges of PCT concentration:

- $<0.5 \mathrm{ng} / \mathrm{ml}$ - low probability of sepsis development;

- $0.5-2.0 \mathrm{ng} / \mathrm{ml}$ - sepsis development is unlikely;

- 2.0-10.0 ng/ml - sepsis development is likely;

$\bullet \geq 10.0 \mathrm{ng} / \mathrm{ml}$ - high probability of sepsis development.

On the basis on these criteria, PCT levels in the patients were determined as follows: PCT $<2.0 \mathrm{ng} / \mathrm{ml}-$ sepsis development is unlikely, PCT level $\geq 2.0 \mathrm{ng} / \mathrm{ml}$ - high probability of sepsis development. The study demonstrated that the PCT level $\geq 2.0 \mathrm{ng} / \mathrm{ml}$ in infants in the postoperative stage increases the risk of sepsis development 5.6 times $\left(\chi^{2}=5.7 ; p=0.019\right.$; $\mathrm{OR}=5.6 ; 95 \% \mathrm{CI}-1.3-23.9 ;$ tb. 4). Sensitivity and specificity of the procalcitonin test for diagnosing sepsis amounted to $65 \%$ and $67 \%$ respectively. At the same time, negative and positive prognostic value of PCT amounted to $76 \%$ and $63 \%$, respectively.

ROC analysis of the analyzed qualitative laboratory markers of bacterial inflammation (CD64 index and CRP) demonstrated that the area under curve (AUC) for CD64 index in the patients with sepsis amounted to 0.895 with the cutoff point of 2.00 , sensitivity $80 \%$, specificity of $90 \%$ $(\mathrm{p}=0.003)$ and $\mathrm{OR}=9.4(95 \% \mathrm{CI}-1.92-46.35)$. At the same time, the respective negative and positive prognostic value of the CD64 index amounted to $74 \%$ and $77 \%$ (tb. 5, pic. 1).

Recent immunological studies led to the discovery of new potential markers that could be instrumental in diagnosing bacterial sepsis. These markers could be roughly subdivided into 3 groups: cell membrane antigens, cytokines and acute phase proteins [17]. In this study, white blood CD64 index reflecting the CD64 expression level on neutrophil membrane was compared to procalcitonin and C-reactive protein - indicators that are frequently used in cardiac surgical practice. The analysis revealed that the CD64 index is a good early marker of sepsis with high sensitivity (80\%) and specificity (90\%), AUC - 0.895 (0.753-1.000) and cutoff point 2.00; this corresponds to the results of other studies of neonates and infants at multi-profile children's units $[17,18]$. For instance, in a study carried out on patients at the multi-profile pediatric intensive care unit, the highly informative values of the CD64 index were 2.38 or higher for infants (aging from 28 days to 12 months) and 1.86 or higher for neonates (respective AUC values amounted to 0.88 and 0.82 , sensitivity values - to 71 and $77 \%$, specificity values - to 100 and $79 \%$, positive prognostic value -100 and $53 \%$, negative prognostic value - 96 and 92\%) [17]. We may assume that increase in the sample size will result in total correlation of the cutoff points for patients from different age group with the results of the aforementioned study. Impact of septic etiology on levels of bacterial inflammation markers ought not to be negated as well. In the aforementioned study, gram-positive flora prevailed in all the cases of confirmed sepsis [17]. In our group of patients, both gram-positive and gram-negative causative agents were observed in the positive hemoculture. Analysis of impact of the etiologically significant causative agents of sepsis on bacterial inflammation markers may be reasonable if the sample size is larger. 
Use of such routine sepsis markers like procalcitonin and CRP is being discussed in the scientific circles and is considered controversial. Davidson et al. demonstrated that CRP cannot serve as a sepsis marker in the postoperative period in the infants who had undergone cardiosurgical intervention using cardiac bypass surgery, since its level remains elevated within 72 hours after the intervention [19]. This fact is confirmed by our study as well, as no statistically significant differences between the CRP levels in two groups of patients with and without sepsis in postoperative period were observed.

The same study demonstrated that the procalcitonin level may be elevated in the first 24 hours after cardiosurgical interventions; after 24 hours it starts to decrease, therefore it could be used as an early marker of an infection process (24 hours after the operation or later) [19]. It was also proved that the abnormally high PCT level 72 hours after the surgery is independently associated with high need in circulatory support and duration of artificial pulmonary ventilation.

The aforementioned facts compel us to search for new laboratory markers that could predict onset of a generalized infection with high probability rate. Cytokines are under constant scrutiny, e.g., interleukin 6 as a sepsis predictor in infants $[18,20]$ and lipopolysaccharide-binding protein (LPB), which is a promising marker of the early stage of sepsis [21]. A large amount of studies is dedicated to the information value of combinations of different markers aimed at increasing the prognostic value of indicators, e.g., LPB + CD64 index, or total neutrophil count; mature and immature neutrophil ratio + CD64 index [21-23].

Currently, the information value of different early markers of sepsis is rather questionable. On the one hand, clinical diagnosis of sepsis without any microbiological proof is very subjective and depends on clinical course of the postoperative period as well as laboratory data. On the other hand, improvement of condition of all the study subjects with SIRS clinical pattern and no diagnosed source of infection after appropriate antibacterial treatment does not necessarily point to the fact that there were no cases of non-bacterial or non-fungal sepsis in this group unless objective (microbiological) confirmation was obtained. According to several scientists, pathogens may be represented not only by bacteria and fungi, but also by viruses [23]. Therefore, the search for septic markers inevitably leads to another widely discussed question: "What is sepsis?"

It is important to point out that this study is the first of its kind carried out on the infants who have undergone cardiac bypass surgery to treat congenital heart disorders. There are no other published data on prognostic value of the CD64 index in the represented category of patients. The obtained results enables us to consider the CD64 index as one of the earliest and most specific laboratory tests for sepsis diagnosis; it has to be admitted, however, that more substantial studies involving more patients are required.

\section{CONCLUSION}

This study revealed that C-reactive protein cannot be considered a reliable early marker of sepsis, probably due to peculiarities of its dynamics in the postoperative period. Specificity of this indicator is low and amounts to only 50\%. Information value of the procalcitonin test in our study was also lower than the CD64 index. Procalcitonin test has its limitations as a tool for diagnosing sepsis within the first days of life as well as within 24 hours after cardiosurgical interventions using cardiac bypass surgery. This study demonstrated that the CD64 index over 2.00 indicates a higher risk of sepsis development in neonates in the postoperative period. It is reasonable to carry out an additional study of information value of this new bacterial inflammation marker for its subsequent introduction into the routine clinical practice.

\section{REFERENCES}

1. Bizzarro M. J., Raskind C., Baltimore R. S., Gallagher P. G. Seventyfive years of neonatal sepsis at Yale: 1928-2003. Pediatrics. 2005 Sep; 116 (3): 595-602. 
2. The UN Inter-agency Group for Child Mortality Estimation (IGME), Levels and trends in Child Mortality: Report, UNICEF. New York. 2013. Available at:

http://www.childinfo.org/files/Child Mortality Report 2013.pdf

3. Strunin O. V., Gorbatyh Ju. N., Shun'kin A. V., Novikova M. A. Features an answer for the correction of congenital heart defects in infants under extracorporeal circulation. Patologiya krovoobrashcheniya i kardiokhirurgiya - Pathology of vascular system and cardiosurgery. 2009; 1: $55-58$.

4. Wheeler D. S., Jeffries H. E., Zimmerman J. J., Wong H. R., Carcillo J. A. Sepsis in the pediatric cardiac intensive care unit. World J Pediatr Congenit Heart Surg. 2011 Jul 1; 2 (3): 393-9.

5. Goldstein B., Giroir B., Randolph A. International consensus conference on pediatric sepsis: international pediatric sepsis consensus conference: definitions for sepsis and organ dysfunction in pediatrics. Pediatr Crit Care Med. 2005; 6: 2-8.

6. Davis B. H. Improved diagnostic approaches to infection/sepsis detection. Expert Rev Mol Diagn. 2005; 5: 193-207.

7. Ng P. C., Lam H. S. Diagnostical markers for neonatal sepsis. Curr Opin Pediatr. 2006; 18: $125-131$.

8. Casado-Flores J., Blanco-Quiros A., Asensio J., Arranz E., Garrote J. A., Nieto M. Serum procalcitonin in children with suspected sesis: a comparison with C-reactive protein and neutrophil count. Pediatric Critical Care Med. 2003; 4: 190-195.

9. Goia C. Procalcitonin in preterm infants during the first few days of life: introducing an age related monogram. Arch Dis Child Fetal Neonatal Ed. 2006; 91: F283-F286.

10. Livaditi O., Kotanidou A., Psarra A., Dimopoulou I., Sotiropoulou C., Augustatou K. et al. Neutrophil CD64 expression and serum IL-8: sensitive early markers of severity and outcome in sepsis. Cytokine. 2006; 36: 283-290.

11. Davis B. H., Olsen S. H., Ahmad E., Bigelow N. C. Neutrophil CD64 is an improved indicator of infection or sepsis in emergency department patients. Arch Pathol Lab Med. 2006; 130: 654-661.

12. Davis B. H., Bigelow N. C. Comparison of neutrophil CD64 expression, manual myeloid immaturity counts, and automated hematology analyzer flags as indicators of infection or sepsis. Lab Hematol. 2005; 11: 137-147.

13. Nuutila J., Hohenthal U., Laitinen I., Kotilainen P., Rajamaki A., Nikoskelainen J. et al. Simultaneous quantitative analysis of Fc-gamma-RI (CD64) expression on neutrophils and monocytes: a new, improved way to detect infections. J Immunol Methods. 2007; 328: 189-200. 14. Ng P. C., Li G., Chui K. M., Chu W. C., Li K., Wong R. P. et al. Neutrophil CD64 is a sensitive diagnostic marker for early-onset neonatal infection. Pediatr Res. 2004; 56: 796-803. 15. Layseca-Espinosa E., Perez-Gonzalez L. F., Torres-Montes A., Baranda L., de la Fuente H., Rosenstein Y. et al. Expression of CD64 as a potential marker of neonatal sepsis. Pediatr Allergy Immunol. 2002; 13: 319-327.

16. Bhandari V., Wang C., Rinder C., Rinder H. Hematologic profile of sepsis in neonates: neutrophil CD64 as a diagnostic marker. Pediatrics. 2008; 121: 129-134.

17. Mojca Groselj-Grenc, Alojz Ihan, Maja Pavcnik-Arnol, Andreja Natasa Kopitar, Tanja Gmeiner-Stopar et al. Neutrophil and monocyte CD64 indexes, lipopolysaccharide-binding protein, procalcitonin and C-reactive protein in sepsis of critically ill neonates and children. Intensive Care Med. 2009; 35: 1950-1958.

18. Bhandari V. Cytokines in neonatal sepsis: diagnostic and therapeutic considerations. In: Singhi S. ed. Current Concepts in Pediatric Intensive Care. Chandigarh, India: Relume Printec. 2000. P. 92-107.

19. Davidson J., Tong S., Hauck A., Lawson D. S., da Cruz E., Kaufman J. Kinetics of procalcitonin and $\mathrm{C}$-reactive protein and the relationship to postoperative infection in young infants undergoing cardiovascular surgery. Pediatric Research. 2013; 74 (4): 413-419. 
20. Gonzalez B. E., Mercado C. K., Johnson L., Brodsky N. L., Bhandari V. Early markers of late-onset sepsis in premature neonates: clinical, hematological and cytokine profile. J Perinat Med. 2003; 31: 60-68.

21. Romagnoli C., Frezza S., Cingolani A. et al. Plasma levels of interleukin-6 and interleukin10 in preterm neonates evaluated for sepsis. Eur J Pediatr. 2001; 160: 345-350.

22. Tepaev R.F. Use of polyclonal immunoglobulins in the treatment of sepsis. Pediatricheskaya farmakologiya - Pediatric pharmacology. 2011; 8 (3): 19-23.

23. Luyt C. E. Viral disease in ICU patients: a long time underestimated; but aware of overestimation. Intensive Care Med. 2006; 32: 968-970.

Table 1. Nosologic classification of the study subjects $(n=36)$

\begin{tabular}{|l|c|c|c|}
\hline \multicolumn{1}{|c|}{ Congenital heart disorder } & Total & $\begin{array}{c}\text { Patients without } \\
\text { sepsis, } \mathbf{n = 2 0}\end{array}$ & $\begin{array}{c}\text { Patients with } \\
\text { sepsis, n= 16 }\end{array}$ \\
\hline TGA & 6 & 3 & 3 \\
\hline $\begin{array}{l}\text { CoA + aortic arch hypoplasia }+ \\
\text { ventricular septal defect }\end{array}$ & 8 & 4 & 4 \\
\hline ToF & 4 & 4 & 2 \\
\hline $\begin{array}{l}\text { Pulmonary atresia + ventricular septal } \\
\text { defect }\end{array}$ & 2 & 0 & 0 \\
\hline Common truncus arteriosus & 2 & 2 & 2 \\
\hline Hypoplastic left heart syndrome & 3 & 1 & 1 \\
\hline DORV + TGA & 2 & 1 & 1 \\
\hline $\begin{array}{l}\text { Univentricular heart + TGA + } \\
\text { pulmonary valve atresia }\end{array}$ & 1 & 1 & 1 \\
\hline $\begin{array}{l}\text { Univentricular heart + TGA + aortic } \\
\text { arch hypoplasia }\end{array}$ & 1 & 0 & 1 \\
\hline $\begin{array}{l}\text { PAPVD + atrial septal defect + tricuspid } \\
\text { insufficiency }\end{array}$ & 1 & 0 & 1 \\
\hline $\begin{array}{l}\text { Ventricular septal defect }+ \text { atrial septal } \\
\text { defect }\end{array}$ & 3 & 2 & 0 \\
\hline Atrial septal defect + high PH & 2 & 1 & 1 \\
\hline Ventricular septal defect & 1 & 1 & \\
\hline
\end{tabular}

Note. TGA - transposition of the great arteries, CoA - aortic coarctation, ToF - tetralogy of Fallot, DORV - double-outlet right ventricle, PAPVD - partial anomalous pulmonary valve disorder, $\mathrm{PH}$ - pulmonary hypertension. 
Table 2. SIRS criteria in children [5]

\section{Presence of at least two of the following symptoms, one of which is mandatorily either} temperature change or abnormally high white blood count

- Central body temperature (measured rectally, orally, in bladder or via central catheter) $>38.5$ or $<36^{\circ} \mathrm{C}$.

- Tachycardia, defined as the heart rate with more than 2 standard deviations from the age-normal values in the absence of external stimuli, medications or pain stimuli; or inexplicably high heart rate for the period ranging from 30 minutes to 4 hours; or (for infants) bradycardia defined as the heart rate below the $10^{\text {th }}$ percentile in the absence of external stimuli of the vagus nerve, use of beta blockers or congenital heart disorders; or, in other words, inexplicable decrease in the heart rate for 30 minutes.

- Tachypnea, defined as the mean respiratory frequency over the $90^{\text {th }}$ percentile of the age-normal values, or need in mechanical ventilation not associated with an acute process, neuromuscular disorder or use of narcotic analgesics.

- White blood count over or below the age norm (excluding secondary decrease in the white blood count due to the use of chemotherapy to treat leukemia), or more than $10 \%$ of neocytes.

Table 3. Clinical and lab profile of patients with or without sepsis $(n=36)$

\begin{tabular}{|l|c|c|c|}
\hline \multicolumn{1}{|c|}{ Clinical and laboratory profile } & $\begin{array}{c}\text { Patients without sepsis, } \\
\mathbf{n = 2 0}\end{array}$ & $\begin{array}{c}\text { Patients with sepsis, } \\
\mathbf{n = 1 6}\end{array}$ & $\mathbf{p}$ \\
\hline Age (in months) & $4.5(1.74-7.5)$ & $2.0(0.3-11.4)$ & 0.789 \\
\hline Cardiac bypass duration (in minutes) & $92(65-134)$ & $126(93-155)$ & 0.128 \\
\hline $\begin{array}{l}\text { Duration of aortic occlusion (in } \\
\text { minutes) }\end{array}$ & $41(28-68)$ & $55(37-76)$ & 0.290 \\
\hline Duration of RICU stay (in days) & $4.75(2.2-9.5)$ & $10.0(6.1-23.0)$ & 0.039 \\
\hline $\begin{array}{l}\text { Duration of inotropic support (in } \\
\text { days) }\end{array}$ & $2.75(0.65-5.5)$ & $8.75(4.1-19.0)$ & 0.008 \\
\hline Duration of APV (in days) & $1.4(0.9-3.5)$ & $19.0(5.25-35.25)$ & 0.004 \\
\hline Mortality rate, patients (\%) & $2(10)$ & $8(50)$ & 0.011 \\
\hline $\begin{array}{l}\text { White blood count (CBC) (in } \\
\text { thousands per mcl) }\end{array}$ & $12.05(9.0-13.9)$ & $13.5(9.1-17.1)$ & 0.404 \\
\hline CD64 index & $1.23(1.05-1.88)$ & $2.29(1.96-3.32)$ & 0.001 \\
\hline CRP (in mg/l) & $27.08(13.18-42.08)$ & $41.01(22.24-91.51)$ & 0.123 \\
\hline
\end{tabular}

Note. RICU - resuscitation and intensive care unit, APV - artificial pulmonary ventilation, CBC - complete blood count, CRP - C-reactive protein. Quantitative variables are represented by medians (quartiles).

Table 4. Amount of patients with or without sepsis with different procalcitonin (PCT) levels

\begin{tabular}{|c|c|c|c|}
\hline PCT level & $\begin{array}{c}\text { Patients without sepsis, } \mathbf{n}=\mathbf{2 0}, \\
\text { abs. (\%) }\end{array}$ & $\begin{array}{c}\text { Patients with sepsis, } \mathbf{n}=\mathbf{1 6}, \\
\text { abs. (\%) }\end{array}$ & p \\
\cline { 1 - 3 }$<2,0 \mathrm{ng} / \mathrm{ml}$ & $13(65)$ & $4(25)$ & \multirow{2}{*}{0,019} \\
\hline PCT $\geq 2,0 \mathrm{ng} / \mathrm{ml}$ & $7(35)$ & $12(75)$ & \\
\hline
\end{tabular}


Table 5. Description of the ROC curve. Possibility of using laboratory markers for diagnosing sepsis in neonates in the postoperative period: results of a ROC analysis.

\begin{tabular}{|l|c|c|c|c|c|}
\hline \multicolumn{1}{|c|}{ Index } & Cutoff point & AUC (95\% CI) & $\mathbf{p}$ & $\begin{array}{c}\text { Sensitivity, } \\
\text { specificity, \% }\end{array}$ & OR (95\% CI) \\
\hline CD64 index & $>2.00$ & $\begin{array}{c}0.895 \\
(0.753-1.000)\end{array}$ & 0.003 & $\begin{array}{c}80(47.6-92.7) \\
90(50.9-91.3)\end{array}$ & $9.4(1.92-46.35)$ \\
\hline CRP & $\mathrm{ns}$ & $\begin{array}{c}0.720 \\
(0.488-0.952)\end{array}$ & 0.096 & $\begin{array}{c}100(69.2-100) \\
50(21.1-78.9)\end{array}$ & $\mathrm{ns}$ \\
\hline
\end{tabular}

Note. AUC - area under curve, CI - confidence interval, OR - odds ratio, ns - not significant (statistically).

Picture. ROC curves for the laboratory parameters under study: CD64 index and C-reactive protein level.

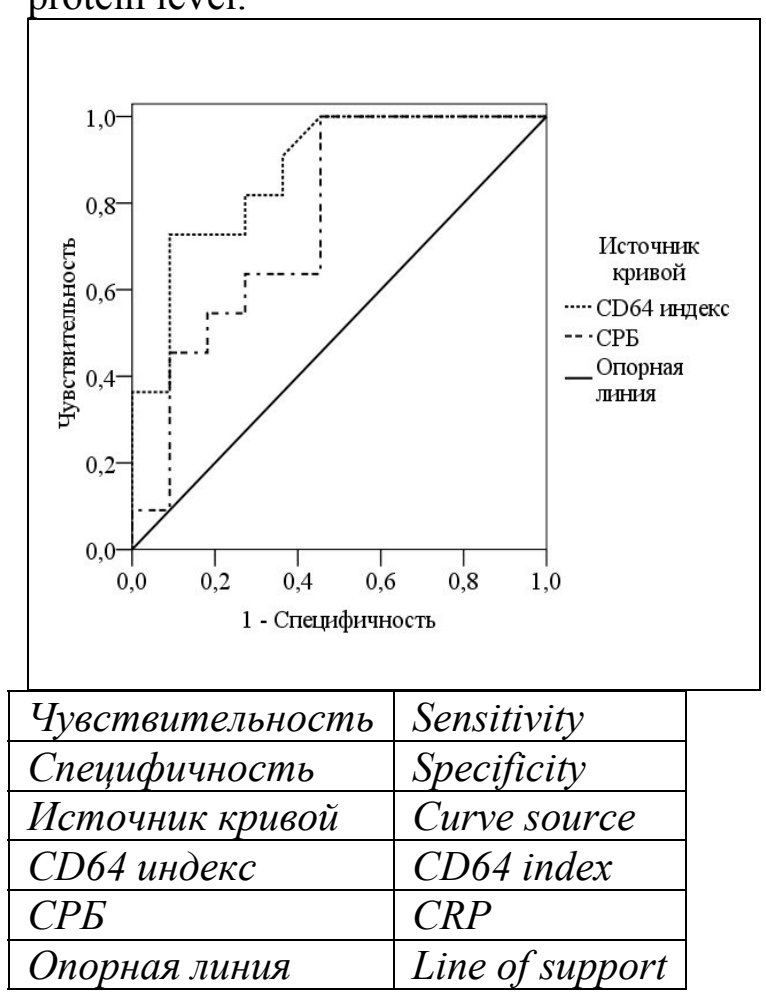

